\title{
miRNA-146a induces vascular smooth muscle cell apoptosis in a rat model of coronary heart disease via NF-kB pathway
}

\author{
Z.W. Wu ${ }^{1,3 *}$, Y.F. $\mathrm{Liu}^{2}$, S. Wang ${ }^{3}$ and B. $\mathrm{Li}^{3}$ \\ ${ }^{1}$ Department of Cardiology, Southern Medical University, \\ Hainan Province People's Hospital, Guangzhou, Guangdong Province, China \\ 2Department of Cardiology, Zhujiang Hospital, Southern Medical University, \\ Guangzhou, Guangdong Province, China \\ ${ }^{3}$ Department of Cardiology, \\ Hainan Western Central Hospital, Danzhou, Hainan Province, China \\ Corresponding author: Z.W. Wu \\ E-mail: nanchegnshou15@163.com
}

Genet. Mol. Res. 14 (4): 18703-18712 (2015)

Received July 15, 2015

Accepted October 2, 2015

Published December 28, 2015

DOI http://dx.doi.org/10.4238/2015.December.28.19

ABSTRACT. The aim of this study was to investigate the role of miRNA$146 a$ in modulating the function of vascular smooth muscle cells in a rat model of coronary heart disease. Vascular smooth muscle cells were isolated and cultured from the rat coronary heart disease model and normal rats (controls). miRNA-146a levels were measured in vascular smooth muscle cells obtained from rats with coronary heart disease and control rats. The proliferation, growth, apoptosis, and activation of the NF-KB pathway in the vascular smooth muscle cells were detected using the MTT assay and flow cytometry, respectively. The role of the NF-KB pathway in modulating the apoptosis of vascular smooth muscle cells was investigated by measuring the reactivity of the cells to an NF-KB pathway inhibitor (TPCA-1). Vascular smooth muscle cells from the disease model exhibited higher levels of miRNA-146a than that by the normal controls $(P=0.0024)$. The vascular smooth muscle cells obtained from rats with 
coronary heart disease showed decreased proliferation and growth and increased apoptosis. miRNA-146a overexpression elevated the rate of cell apoptosis. The NF-kB pathway was activated in vascular smooth muscle cells obtained from rats with coronary heart disease. Inhibition of the NF$\mathrm{KB}$ pathway significantly decreased the rate of vascular smooth muscle cell apoptosis in coronary heart disease rats $(P=0.0038)$. In conclusion, miRNA146 a was found to induce vascular smooth muscle cell apoptosis in rats with coronary heart disease via the activation of the NF-kB signal pathway.

Key words: miRNA-146a; NF-KB signal pathway; Cell apoptosis; Coronary heart disease rat model; Vascular smooth muscle cells

\section{INTRODUCTION}

The incidence of cardio- and cerebro-vascular diseases, specifically coronary heart disease, has increased with the enhanced quality of life and changes in life style, which has adversely affected public health (Cases and Mestres, 2009; Eriksen et al., 2015; Muneera et al., 2015). Traditional therapies for cardiovascular disease are generally non-specific, and can cause complications. This necessitates the discovery of specific targets for clinical treatment (Cases and Mestres, 2009; Pang et al., 2015; Ramirez-Ramirez et al., 2015; Sun et al., 2015).

MicroRNA (miRNA) are small, non-coding, double-stranded RNA molecules, approximately 20-25 bp long. miRNA modulate various biological functions, such as cell proliferation, growth, apoptosis, and signal transduction (Chen et al., 2015; Femminella et al., 2015; Higuchi et al., 2015; Wang et al., 2015). Previous studies have also reported that miRNA-146a mediates cellular growth and proliferation in various cells (Alexandrov et al., 2014; Xie et al., 2014; Gu et al., 2015; Hill et al., 2015; Tang et al., 2015; Zhou et al., 2015), however, the role of miRNA-146a in coronary heart disease remains to be elucidated. Therefore, this study was designed to investigate the modulating effect of miRNA-146a on vascular smooth muscle cells from a coronary heart disease rat model.

The nuclear factor - kappa B (NF-kB) pathway plays an important role in cellular growth, proliferation, and signal transduction. Previous studies have indicated that heart disease results in the activation of the NF-KB pathway in, and abnormal growth or proliferation of, vascular smooth muscle cells; however, the underlying molecular mechanisms remain unclear (Burkitt et al., 2015; Cheng et al., 2015; Lee et al., 2015; Tsubaki et al., 2015; Xi et al., 2015). We also attempted to determine the role of the NF-KB pathway in the vascular smooth muscle cells of a coronary heart disease rat model, and its related molecular mechanism.

In this study, a rat model of coronary heart disease was used to investigate the role of miRNA-146a in modulating vascular smooth muscle cells.

\section{MATERIAL AND METHODS}

\section{Reagents and antibodies}

Caspase- 3 and Caspase- 8 activity assay kits were purchased from Biyuntian Biology 
Technology Institute (Haimen, China). 3-(4,5-dimethylthiazol-2-yl)-2,5-diphenyltetrazolium bromide (MTT), tetramethylrhodamine ethyl ester (TMRE), and fluorescein isothiocyanate (FITC)Annexin-V were obtained from Sigma-Aldrich (St. Louis, MO, USA). Anti-rat-NF-kB antibody, an inhibitor of the NF-KB pathway (TPCA-1), Dulbecco's modified Eagle's medium (DMEM), and fetal bovine serum (FBS) were also purchased from Sigma-Aldrich. The reverse transriptase polymerase chain reaction (RT-PCR) reagents were purchased from Dingguo Biology Technology Inc. (Beijing, China).

\section{Modeling of rat coronary heart disease}

Male SD rats (8 weeks) were purchased from the Hufu Animal Center (Beijing, China). The coronary heart disease rat model was developed using a method detailed in a previously published paper (Hwee et al., 2015). In brief, coronary heart disease was induced by feeding the SD rats with a high-fat diet, consisting of $87.3 \%$ standard chow, $0.49 \%$ sodium cholate hydrate, $0.21 \%$ propylthiouracil, $10 \%$ lard, and $2 \%$ cholesterol for 8 weeks; the rat models were identified based on the coronary heart disease guide. Rats in the control groups were fed with standard chow. Each group consisted of 48 rats.

These rats were used in all subsequent experiments; all procedures were approved by the Animal Ethics Committee of the Hainan Province People's Hospital.

\section{Isolation and culture of vascular smooth muscle cells}

Vascular smooth muscle cells were isolated from rats with and without (normal control) coronary heart disease using a protocol detailed in a previous study (Hwee et al., 2015). The rats were anesthetized using 5\% avertin; the hearts and aorta were removed from the anesthetized rats and placed in $200 \mathrm{U} / \mathrm{mL}$ Hanks salt solution supplemented with $200 \mathrm{mg} / \mathrm{mL}$ streptomycin. Adipose tissue was removed from the hearts and aorta; subsequently, these were rinsed in DMEM, and cut into small pieces. The supernatant was collected and the samples allowed to set for $6 \mathrm{~min}$. This process was repeated thrice. The small tissue pieces and the cell suspension were then cultured in a cell culture flask. The isolated cells were re-suspended in DMEM and cultured at $37^{\circ} \mathrm{C}$ in a $5 \% \mathrm{CO}_{2}$ incubator.

\section{RT-PCR}

RNA was extracted from vascular smooth muscle cells as per a previously described protocol (Hwee et al., 2015). The samples were amplified using the following primers: miRNA-146a (5'-3'), R-GGTTACCTTGTTACGACTT and F-AGAGTTTGATCCTGGCTCA; and GAPDH (to normalize the miRNA-146a expression), (5'-3') F-CGGAGTCAACGGATTTGGTCGTAT, R-AGCCTTC TCCATGGTGGTGAAGAC. The PCR products were electrophoresed on an agarose gel to detect the level of miRNA-146a expression in vascular smooth muscle cells obtained from normal rats and rats with coronary heart disease.

\section{MTT assay}

The cell viability of cultured vascular smooth muscle cells was determined by the MTT assay, as described in a previous report (Hwee et al., 2015). In brief, the cells were cultured in 
96-well plates $(10,000 /$ well) for 3 days prior to the addition of MTT. These plates were further incubated for $4 \mathrm{~h}$; subsequently, dimethyl sulfoxide (DMSO; $160 \mu \mathrm{L}$ ) was added to each well, and the plates were vortexed for $8 \mathrm{~min}$. The optical density (OD) at $490 \mathrm{~nm}$ was measured using a micro-plate reader. The growth chart was mapped with OD on the $\mathrm{Y}$ axis and time interval as the $X$ axis. In the analysis of the pathway, the isolated vascular smooth muscle cells were pre-treated with the NF-KB pathway inhibitor (TPCA-1) for $24 \mathrm{~h}$; the cell viability was subsequently detected using the MTT method.

\section{Flow cytometry analysis of PS exposure}

Apoptosis in cultured vascular smooth muscle cells was determined by flow cytometry as described in a previous report (Hwee et al., 2015). The cultured cells were collected and suspended ( $8 \times 10^{4}$ cells) with $250 \mu \mathrm{L}$ Annexin-V-FITC binding solution. The Annexin-V-FITC reaction solution $(5 \mu \mathrm{L})$ was subsequently added, and the samples incubated for 20 min in the dark. Apoptosis was determined in a flow cytometer at excitation and emission wavelengths of $488 \mathrm{~nm}$ and $625 \mathrm{~nm}$, respectively.

\section{Measurement of the caspase- 3 activity}

The caspase- 3 activity of cultured vascular smooth muscle cells was determined using a caspase- 3 activity assay kit, as described in a previous study (Hwee et al., 2015). In brief, the cells were cultured in a 96-well plate (10,000/well), and treated with lysis buffer from the kit for $10 \mathrm{~min}$. The supernatant was then collected and analyzed in a micro-plate reader at an OD of $560 \mathrm{~nm}$.

\section{Measurement of the NF-kB signal pathway activity}

The NF-kB signal pathway activity of cultured vascular smooth muscle cells was determined using a commercial assay kit, as described in a previous report (Hwee et al., 2015). The cells were treated with lysis buffer for $30 \mathrm{~min}$ on ice, and subsequently subjected to sodium dodecyl sulfate polyacrylamide gel electrophoresis (SDS-PAGE) and western blot analysis. Anti-NF-kB and anti-actin antibodies were used for these experiments.

\section{Statistical analysis}

All data were analyzed using the SPSS (v.16.0) software platform (IBM, Armonk, NY, USA). All data are presented as mean \pm standard deviation (SD). The different groups were compared by one-way analysis of variance (ANOVA). P-values $\leq 0.05$ and $\leq 0.01$ were considered to be significant and highly significant, respectively.

\section{RESULTS}

\section{Vascular smooth muscle cells from rats with coronary heart disease showed higher miRNA-146a expression than those from normal control rats}

The level of expression of miRNA-146a was measured in the vascular smooth muscle cells obtained from sample and control rats by RT-PCR $(P=0.0024)$. The vascular smooth muscle 
cells extracted from rats with coronary heart disease showed significantly higher levels of miRNA146a than those obtained from normal control rats (Figure 1).

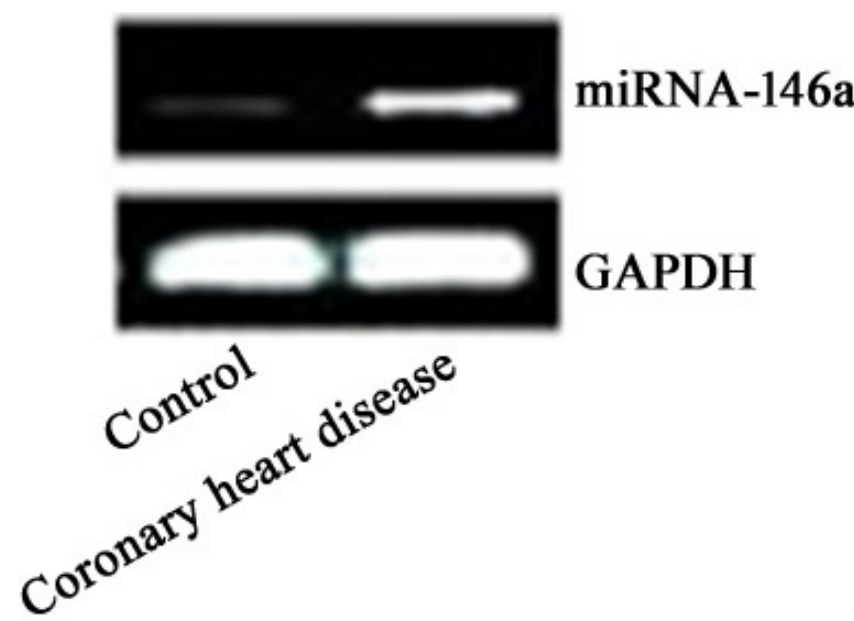

Figure 1. Vascular smooth muscle cells obtained from rats with coronary heart disease showed higher miRNA-146a levels than those from normal control rats.

Rats with coronary heart disease display inhibited vascular smooth muscle cell growth

Rats with coronary heart disease showed significantly lowered proliferation of vascular smooth muscle cells than normal control rats $(P=0.0037$; Figure 2$)$.

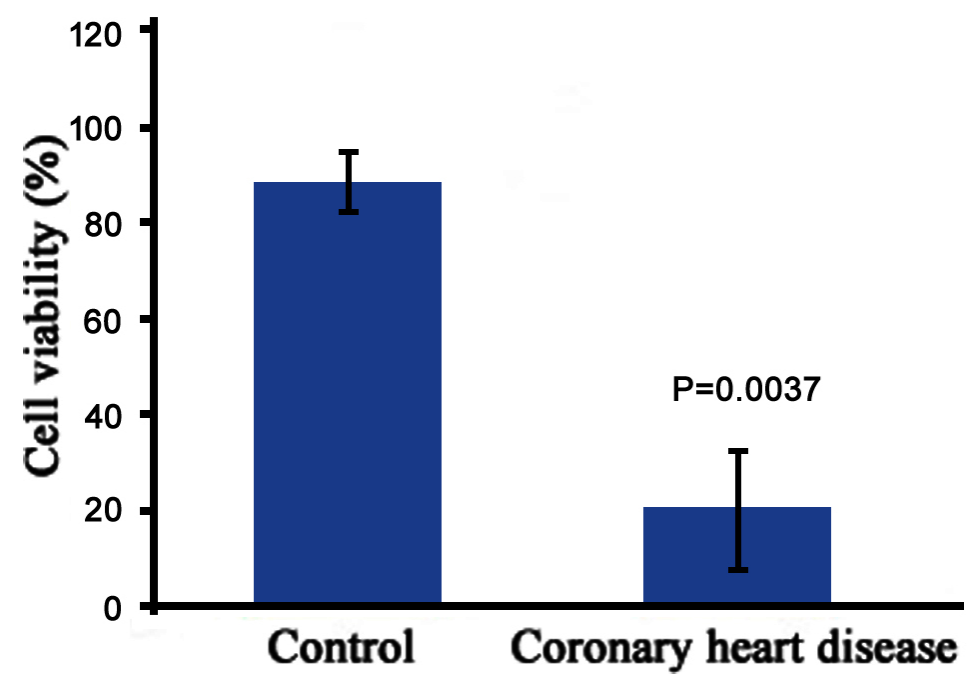

Figure 2. Vascular smooth muscle cell growth was inhibited in rats with coronary heart disease. 


\section{Vascular smooth muscle cells from rats with coronary heart disease undergo increased apoptosis}

The mitochondrial membrane potential of vascular smooth muscle cells extracted from rats with coronary heart disease was significant lower than that in cells obtained from normal control rats. Alternately, vascular smooth muscle cells extracted from rats with coronary heart disease showed a significantly higher PS exposure rate and caspase-3 activity than those from normal control rats (Figures 3,4, and 5). These results suggested an increased rate of vascular smooth muscle cell apoptosis in coronary heart disease rats.

Overexpression of miRNA-146a in vascular smooth muscle cells extracted from rats with coronary heart disease was followed by increased apoptosis (Figure 5), suggesting that vascular smooth muscle cell apoptosis in rats with coronary heart disease was induced by miRNA-146a.

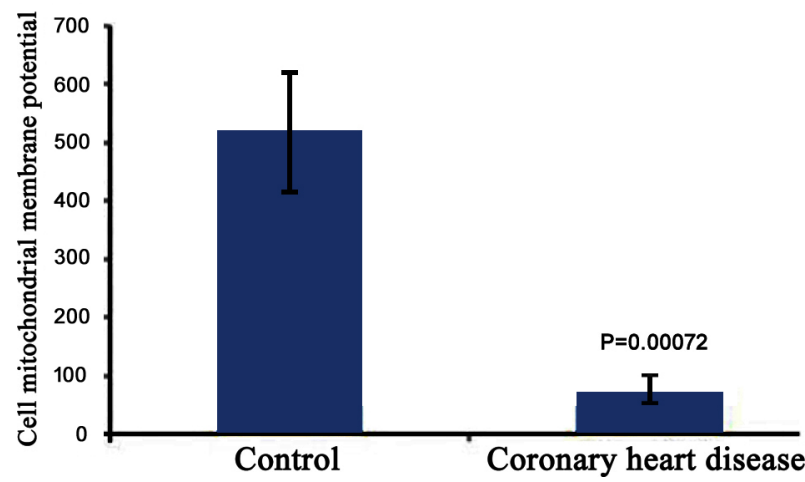

Figure 3. Vascular smooth muscle cells showed decreased mitochondrial membrane potential in the coronary heart disease rat model.

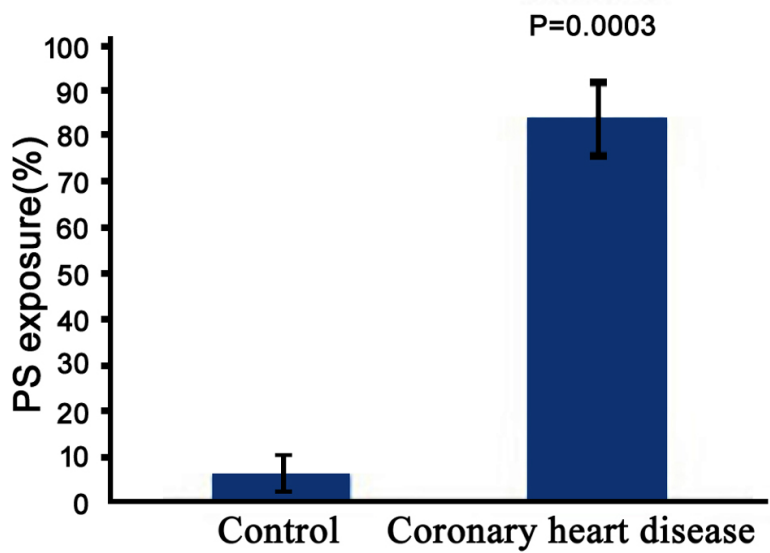

Figure 4. Vascular smooth muscle cells showed increased exposure of phosphatidylserine in the coronary heart disease rat model. 


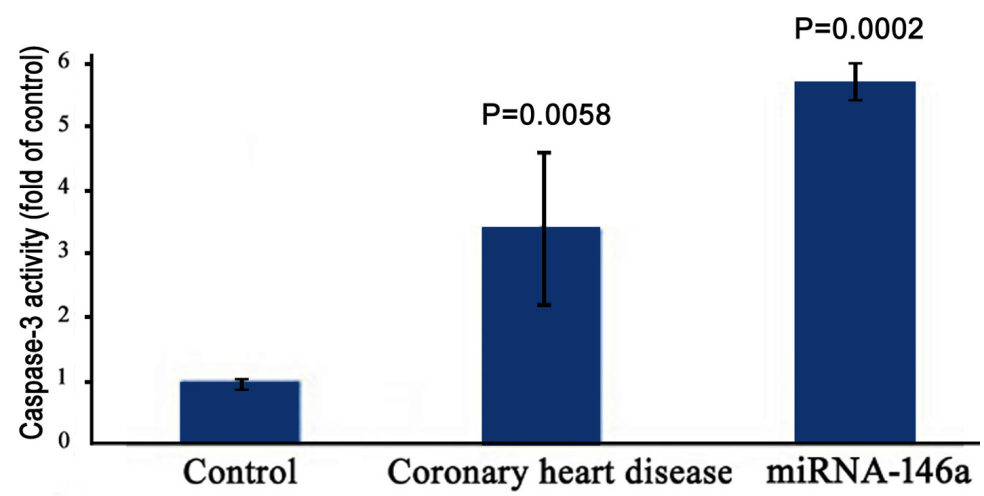

Figure 5. Vascular smooth muscle cells showed increased caspase-3 activation in the coronary heart disease rat model.

\section{Activation of NF-KB in vascular smooth muscle cells from rats with coronary heart disease}

We observed a significant increase in the activation of NF-kB in vascular smooth muscle cells extracted from rats with coronary heart disease compared to those from normal control rats (Figure 6). NF-kB activation was further increased with the over-expression of miRNA-146a, indicating that miRNA-146a might directly or indirectly activate NF-KB.

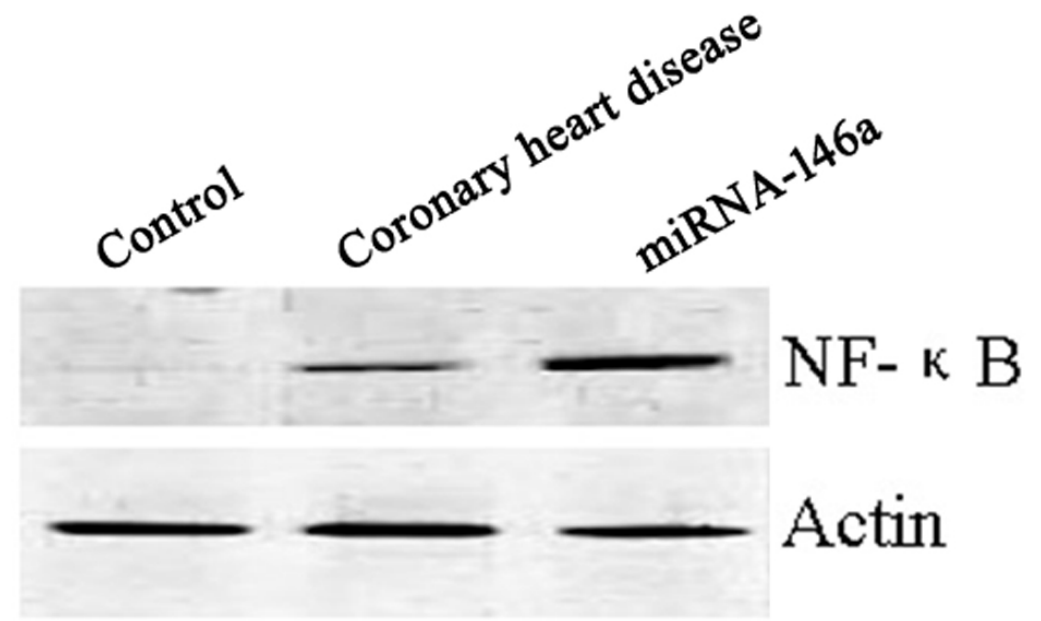

Figure 6. NF-KB pathway was activated in the vascular smooth muscle cells of rats with coronary heart disease.

\section{Vascular smooth muscle cell apoptosis is significantly decreased after NF-kB inhibition in rats with coronary heart disease}

Vascular smooth muscle cell apoptosis in rats with coronary heart disease was significantly decreased after the NF-KB signal pathway was inhibited (Figure 7). This result suggested that apoptosis of the vascular smooth muscle cells extracted from rats with coronary heart disease was mediated by the NF-kB signal pathway. 


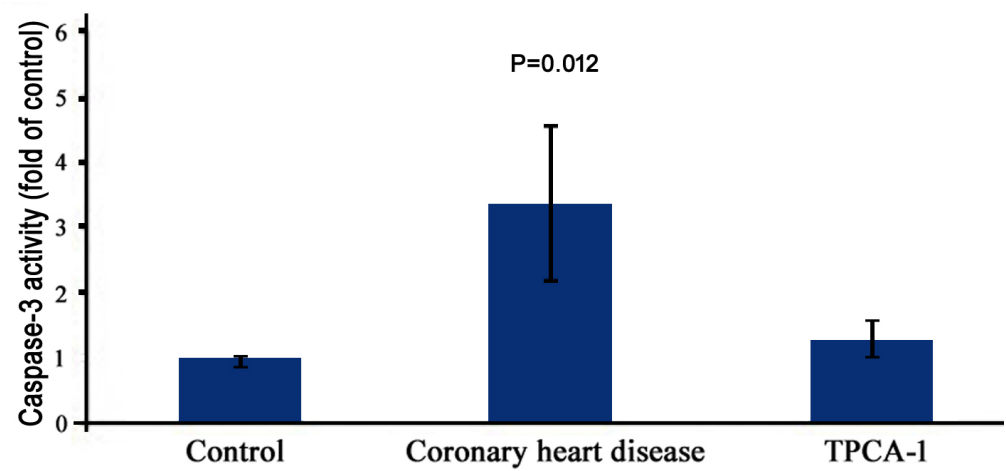

Figure 7. Vascular smooth muscle cell apoptosis was decreased in the coronary heart disease rat model after the NF-kB pathway was inhibited.

\section{DISCUSSION}

In this study, the effects of miRNA-146a on the vascular smooth muscle cells of a coronary heart disease rat model were investigated. Vascular smooth muscle cells from rats with coronary heart disease showed higher levels of miRNA-146a than those from normal control rats. This result suggested that miRNA-146a may play a role in modulating vascular smooth muscle cells. We also attempted to determine the underlying mechanism with which miRNA-146a modulated vascular smooth muscle cells; we observed that vascular smooth muscle cells from rats with coronary heart disease showed a significantly suppressed growth and proliferation rate, and a high rate of apoptosis. miRNA-146a was overexpressed in smooth muscle cells from rats with coronary heart disease to determine if miRNA-146a mediates the apoptosis of smooth muscle cells from coronary heart disease rats. The results revealed an increase in the apoptosis rate post- miRNA-146a overexpression. These results indicated that miRNA-146a mediates the apoptosis of smooth muscle cells in rats with coronary heart disease. This is in agreement with the observations of previous studies (Eriksen et al., 2015; Muneera et al., 2015; Pang et al., 2015; Ramirez-Ramirez et al., 2015; Sun et al., 2015).

Cell apoptosis occurs via two pathways: the death receptor-mediated external signal pathway and the mitochondria-mediated internal signal pathway. The signal pathway of miRNA-146amediated apoptosis was investigated in the vascular smooth muscle cells of rats with coronary heart disease; this resulted in the detection of the activity of caspase-8, an indicator of the death receptor-mediated external signal pathway. We observed that smooth muscle cells extracted from rats with coronary heart disease were not activated. Therefore, we concluded that smooth muscle cell apoptosis in coronary heart disease rats was mediated by the mitochondrial pathway. This was also in agreement with the results of previous studies (Chen et al., 2015; Femminella et al., 2015; Wang et al., 2015).

The molecular mechanism of miRNA-146a-mediated vascular smooth muscle cell apoptosis in rats with coronary heart disease was further investigated by measuring the activity of the protein kinase. The NF-KB signal pathway plays an important role in the growth, proliferation, and signal transduction of different cell types. Previous studies (Burkitt et al., 2015; Cheng et al., 2015; Tsubaki et al., 2015; Xi et al., 2015) have suggested that the NF-KB pathway is activated in vascular smooth muscle cells, and that these cells show abnormal growth and proliferation during the occur- 
rence of heart disease. Therefore, the activation of the NF-kB signal pathway in vascular smooth muscle cells was analyzed. The findings of this study showed that the NF-kB signal pathway was activated in vascular smooth muscle cells obtained from rats with coronary heart disease. In addition, overexpression of miRNA-146a was found to elevate this activation, suggesting that miRNA$146 \mathrm{a}$ activates the NF-KB signal pathway in the vascular smooth muscle cells of a coronary heart disease rat model. The NF-KB signal pathway was blocked to further determine if miRNA-146a mediates the apoptosis of vascular smooth muscle cells in a coronary heart disease rat model through this pathway; the NF-kB signal pathway inhibitor was found to suppress the apoptosis in the vascular smooth muscle cells of a coronary heart disease rat model. Taken together, these results indicated that miRNA-146a mediates the apoptosis of vascular smooth muscle cells in a coronary heart disease rat model through the NF-KB signal pathway. This is consistent with the results of a previous study (Femminella et al., 2015).

This study provides further proof that miRNA-146a plays an important role in modulating the growth, proliferation, and death of vascular smooth muscle cells in a coronary heart disease rat model. The possible role of miRNA-146a in coronary heart disease patients can be further investigated by analyzing the level of miRNA-146a, activation of NF-KB signal pathway, and apoptosis in the vascular smooth muscle cells obtained clinically from these patients. Alternately, the rat coronary heart disease model can be used for this purpose. In future studies, any improvements in coronary heart disease can be measured in an in vivo animal model after altering the miRNA-146a level and NF-kB signal pathway activation.

\section{Conflicts of interest}

The authors declare no conflict of interest.

\section{ACKNOWLEDGMENTS}

We thank the anonymous reviewers for reviewing this manuscript.

\section{REFERENCES}

Alexandrov PN, Dua P and Lukiw WJ (2014). Up-Regulation of miRNA-146a in progressive, age-related inflammatory neurodegenerative disorders of the human CNS. Front Neurol. 5: 181.

Burkitt MD, Hanedi AF, Duckworth CA, Williams JM, et al. (2015). NF-kappaB1, NF-kappaB2 and c-Rel differentially regulate susceptibility to colitis-associated adenoma development in C57BL/6 mice. J. Pathol. 236: 326-336.

Cases M and Mestres J (2009). A chemogenomic approach to drug discovery: focus on cardiovascular diseases. Drug Dicov. Today 14: 479-485.

Chen C, Wang Y, Yang S, Li H, et al. (2015). MiR-320a contributes to atherogenesis by augmenting multiple risk factors and down-regulating SRF. J. Cell Mol. Med. 19: 970-985.

Cheng H, Bo Y, Shen W, Tan J, et al. (2015). Leonurine ameliorates kidney fibrosis via suppressing TGF-beta and NF-kappaB signaling pathway in UUO mice. Int. Immunopharmacol. 25: 406-415.

Eriksen A, Tillin T, O'Connor L, Brage S, et al. (2015). The impact of health behaviours on incident cardiovascular disease in Europeans and South Asians - a prospective analysis in the UK SABRE study. PloS One 10: e0117364.

Femminella GD, Ferrara N and Rengo G (2015). The emerging role of microRNAs in Alzheimer's disease. Front. Physiol. 6: 40.

Gu SX, Li X, Hamilton JL, Chee A, et al. (2015). MicroRNA-146a reduces IL-1 dependent inflammatory responses in the intervertebral disc. Gene 555: 80-87.

Higuchi C, Nakatsuka A, Eguchi J, Teshigawara S, et al. (2015). Identification of circulating miR-101, miR-375 and miR-802 as biomarkers for type 2 diabetes. Metabolism 64: 489-497.

Hill JM, Clement C, Zhao Y and Lukiw WJ (2015). Induction of the pro-inflammatory NF-kB-sensitive miRNA-146a by human neurotrophic viruses. Front. Microbiol. 6: 43. 
Hwee DT, Kennedy AR, Hartman JJ, Ryans J, et al. (2015). The small-molecule fast skeletal troponin activator, CK-2127107, improves exercise tolerance in a rat model of heart failure. J. Pharmacol. Exp. Ther. 353: 159-168.

Lee HS, Kang P, Kim KY and Seol GH (2015). Foeniculum vulgare Mill. protects against lipopolysaccharide-induced acute lung injury in mice through ERK-dependent NF-kappaB activation. Korean J. Physiol. Pharmacol. 19: 183-189.

Muneera K, Majeed A and Naveed AK (2015). Comparative evaluation of Nigella sativa (Kalonji) and simvastatin for the treatment of hyperlipidemia and in the induction of hepatotoxicity. Pak. J. Pharm. Sci. 28: 493-498.

Pang H, Han B, Li ZY and Fu Q (2015). Identification of molecular markers in patients with hypertensive heart disease accompanied with coronary artery disease. Genet. Mol. Res. 14: 93-100.

Ramirez-Ramirez R, Gutierrez-Angulo M, Magana MT, Moreno-Ortiz JM, et al. (2015). Effect of ZNF217 gene polymorphisms on colorectal cancer development in a Mexican population. Genet. Mol. Res. 14: 362-367.

Sun B, Li J, Dong M, Yang L, et al. (2015). Diversity of platelet function and genetic polymorphism in clopidogrel-treated Chinese patients. Genet. Mol. Res. 14: 1434-1442.

Tang Q, Yang Y, Zhao M, Liang G, et al. (2015). Mycophenolic acid upregulates miR-142-3P/5P and miR-146a in lupus CD4+T cells. Lupus 24: 935-942.

Tsubaki M, Takeda T, Ogawa N, Sakamoto K, et al. (2015). Overexpression of survivin via activation of ERK1/2, Akt, and NFkappaB plays a central role in vincristine resistance in multiple myeloma cells. Leuk. Res. 39: 445-452.

Wang CY, Hu LL, Guo MZ, Liu XY, et al. (2015). imDC: an ensemble learning method for imbalanced classification with miRNA data. Genet. Mol. Res. 14: 123-133.

Xi G, Shen X, Wai C, Vilas CK, et al. (2015). Hyperglycemia stimulates p62/PKC $\zeta$ interaction, which mediates NF-кB activation, increased Nox4 expression, and inflammatory cytokine activation in vascular smooth muscle. FASEB J. 29: $4772-4782$.

Xie YF, Shu R, Jiang SY, Song ZC, et al. (2014). miRNA-146 negatively regulates the production of pro-inflammatory cytokines via NF-kappaB signalling in human gingival fibroblasts. J. Inflamm. (Lond) 11: 38.

Zhou X, Zhu J, Zhang H, Zhou G, et al. (2015). Is the microRNA-146a (rs2910164) polymorphism associated with rheumatoid arthritis? Association of microRNA-146a ( $r$ 2910164) polymorphism and rheumatoid arthritis could depend on gender. Joint Bone Spine 82: 166-171. 